\title{
IMPLICAÇÕES QUÍMICAS NA SISTEMÁTICA E FILOGENIA DE Bignoniaceae ${ }^{\#}$
}

Franciane Auxiliadora Cipriani

Museu Nacional, Universidade Federal do Rio de Janeiro, 20940-040 Rio de Janeiro - RJ, Brasil

Maria Raquel Figueiredo

Departamento de Química de Produtos Naturais, Instituto de Tecnologia em Fármacos, Fundação Oswaldo Cruz, Rua Sizenando Nabuco, 100, 21041-250 Rio de Janeiro - RJ, Brasil

Geraldo Luiz Gonçalves Soares

Departamento de Botânica, Instituto de Biociências, Universidade Federal do Rio Grande do Sul, Av. Bento Gonçalves, 9500 , 91501-970 Porto Alegre - RS, Brasil

Maria Auxiliadora Coelho Kaplan*

Núcleo de Pesquisas de Produtos Naturais, Centro de Ciências da Saúde, Bl. H, Universidade Federal do Rio de Janeiro, Cidade Universitária, 21941-590 Rio de Janeiro - RJ, Brasil

Recebido em 6/5/12; aceito em 20/7/12; publicado na web em 5/10/12

\begin{abstract}
CHEMICAL IMPLICATIONS IN SYSTEMATICS AND PHYLOGENY OF Bignoniaceae. Our solemn homage to the great Master Otto R. Gottlieb who knew how to teach the mystery of evolutionary relationships between chemistry and its natural sources. The micromolecular chemical study of the family Bignoniaceae shows a profile predominantly characterized by the occurrence of metabolites derived from acetic acid biosynthetic pathways such as terpenoids, quinones, flavonoids and special aromatic derivatives. Analysis of different chemosystematic parameters for the metabolite data collected, provided valuable information for the systematic characterization of the Bignoniaceae family within the Angiosperm derived taxa.
\end{abstract}

Keywords: Bignoniaceae; chemotaxonomic markers; Angiospermae.

\section{INTRODUÇÃO}

As Bignoniaceae são eudicotiledôneas da ordem Lamiales, onde seus parentes mais próximos não são claros. ${ }^{1-3}$ Apresentam cerca de 827 espécies distribuídas em 82 gêneros. ${ }^{4}$ Esse táxon angiospérmico reúne espécies pantropicais, mas predominantemente neotropicais, com poucos representantes nas regiões temperadas. ${ }^{5}$

Dentro de Lamiales, a família Bignoniaceae está posicionada perto de Avicenniaceae, Lamiaceae, Lentibulariaceae, Phrymaceae, Schlegeliaceae, Verbenaceae e Acanthaceae. ${ }^{6,7}$ Percebe-se, porém, que esse posicionamento não é definitivo devido às mudanças constantes, ainda não totalmente concluídas, na circunscrição das famílias pertencentes à ordem, sendo essas principalmente baseadas em análises macromoleculares e à pequena amostragem estudada.

Atualmente ocorre uma considerável divergência entre autores sobre a classificação infrafamiliar da família Bignoniacae. ${ }^{8,9}$ A classificação mais atual reconhece para a família a sua divisão em oito grandes grupos, sendo eles: Bignonieae, Catalpeae, Jacarandeae, Oroxyleae, Tourrettieae, Tecomeae, Clado Paleotropical e Tabebuia alliance, sendo que alguns gêneros ainda não têm posicionamento definido (Bignoniaceae unassigned to Clades). ${ }^{10}$. Excluindo o gênero Paulownia e a tribo Schlegelieae (antes considerados pertencentes à família Bignoniaceae e agora colocados em Paulowniaceae e Schlegeliaceae), Bignoniaceae é monofilética. ${ }^{11}$

Diversas espécies apresentam importância econômica o que, somado à importância florística dessa família, justifica o aprofundamento do seu conhecimento taxonômico. Nas últimas décadas, as espécies de Bignoniaceae foram extensamente estudadas do ponto de vista fitoquímico. ${ }^{12-19}$ Entretanto, existem poucos trabalhos que

*e-mail: makaplan@uol.com.br

\#Artigo em homenagem ao Prof. Otto R. Gottlieb (31/8/1920-19/6/2011) empregam esse extenso conhecimento químico como ferramenta em estudos taxonômicos. ${ }^{12,20,21}$ Cipriani e colaboradores ${ }^{22}$ determinaram o perfil químico de Bignoniaceae, caracterizado pela ocorrência de terpenoides, quinonas, derivados aromáticos especiais e flavonoides. A biossíntese dos seus metabólitos especiais baseia-se, principalmente, na via do ácido acético, o que está de acordo com o seu posicionamento entre as angiospermas mais derivadas. Entretanto, com a mudança ocorrida na circunscrição da família, percebeu-se a necessidade que esse perfil fosse refeito, já que em nível de tribos, ele não mais correspondia à realidade da família.

Portanto, o emprego da abordagem metodológica da Quimiossistemática Micromolecular vem auxiliar na melhor compreensão das relações filogenéticas nesse importante táxon angiospérmico.

O presente estudo teve como objetivo a elucidação de polarizações evolutivas em Bignoniaceae, através da análise do padrão de ocorrência dos seus marcadores quimiotaxonômicos.

\section{PARTE EXPERIMENTAL}

O levantamento da ocorrência de micromoléculas em espécies de Bignoniaceae foi feito através da consulta às obras de referência, Chemical Abstract Service de 1907 a 2011 e Biological Abstracts de 1960 a 2011. As palavras chave utilizadas nessa pesquisa bibliográfica foram os nomes dos gêneros válidos para as famílias.

Os dados obtidos nessa pesquisa bibliográfica foram digitados e formaram um banco de dados sobre a ocorrência de metabólitos secundários (micromoléculas) em cada grupo considerado. As informações foram distribuídas em Tabelas. A partir da análise desse banco de dados foi elaborado o perfil químico da família, foram determinados os seus marcadores quimiotaxonômicos, isto é, as classes de substâncias de ocorrência mais significativa e com maior variabilidade estrutural, e calculados os seus parâmetros quimiotaxonômicos. 


\section{Número de ocorrências (NO)}

É um parâmetro químico que fornece o grau de relevância de uma determinada categoria metabólica para um táxon escolhido. Representa o número de substâncias, de uma classe química específica, produzidas por determinado táxon.

\section{Percentagem de número de ocorrências (NO\%)}

Para normalizar os dados foi calculada a percentagem do número de ocorrências, na qual o somatório do número de ocorrências de uma determinada classe biogenética multiplicado por 100 é dividido pelo número total de ocorrências registradas.

\section{Índice químico-morfológico (IH)}

Para possibilitar comparações de dados químicos com dados morfológicos foram introduzidos índices representativos desses parâmetros. O índice químico-morfológico ou índice de herbacidade (IH) é baseado no avanço evolutivo morfológico seguindo o hábito predominante das espécies do táxon em estudo. O valor 100 é atribuído aos táxons formados por somente representantes herbáceos, enquanto que o valor 1 é atribuído aos táxons representados exclusivamente por árvores. Valores intermediários são dados aos táxons com predominância de plantas arbóreas, herbáceas e/ou arbustivas. Esse índice revela a tendência de hábito de cada grupo vegetal e não a herbacidade de cada espécie. ${ }^{23} \mathrm{O}$ hábito relativo a cada gênero da família Bignoniaceae foi descrito em 2007, por Lohmann e Ulloa. ${ }^{4}$

\section{Relação flavona/flavonol (fo/fl)}

É calculada a partir da razão do número de ocorrências desses dois tipos flavonoídicos para os táxons em estudo.

\section{Relação chiquimato/acetato}

É calculada a partir da razão do número de ocorrências de sustâncias da rota do ácido chiquímico pelo número de ocorrências de substâncias da rota do ácido acético para os táxons em estudo.

\section{Análise das polarizações filogenéticas dos marcadores químicos} em Bignoniaceae

Verificou-se como os caracteres químicos distribuem-se nos gêneros de Bignoniaceae, segundo o esquema filogenético proposto para a família. ${ }^{10}$ Esse tipo de associação entre a metodologia quimiossistemática e a taxonomia molecular permite um alto grau de previsão na ocorrência de dados químicos, o que constitui uma ferramenta valiosa para estudos evolutivos, ecológicos e farmacológicos envolvendo espécies de Bignoniaceae.

\section{RESULTADOS E DISCUSSÃO}

\section{Perfil químico geral da família Bignoniaceae}

O levantamento das informações sobre a biologia molecular da família Bignoniaceae permite afirmar que esse táxon se caracteriza quimicamente pela ocorrência de terpenoides $(\mathrm{NO}=319)$, quinonas $(\mathrm{NO}=151)$, derivados não nitrogenados de cadeia longa da via do acido acético $(\mathrm{NO}=37)$, alcaloides $(\mathrm{NO}=38)$, derivados aromáticos especiais $(\mathrm{NO}=194)$ e flavonoides $(\mathrm{NO}=105)$, entre outros (Figura 1$)$.

A biossíntese de metabólitos secundários na família Bignoniaceae mostra destaque na produção de metabólitos derivados da via

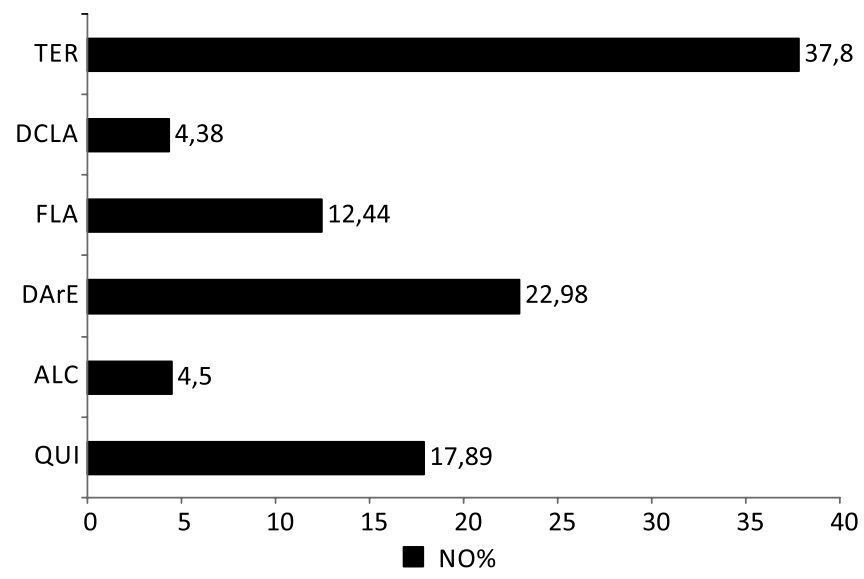

Figura 1. Percentagens dos números de ocorrência (NO\%) de metabólitos especiais encontrados em Bignoniaceae. QUI, Quinonas (NO =151); ALC, Alcaloides $(N O=38) ;$ DArE, Derivados aromáticos especiais $(N O=194)$; FLA, Flavonoides (NO = 105); DCLA, Derivados de cadeia longa da via do acetato $(N O=37)$ e TER, Terpenoides $(N O=319)$

acetato-mevalonato, com a ocorrência de terpenoides. Com esses dados pode-se obter a relação chiquimato/acetato igual a 0,36 para a produção metabólica de Bignoniaceae.

Esse fato está de acordo com o posicionamento das Bignoniaceae entre as Asteridae de Cronquist ${ }^{24}$ como um táxon que explora preferencialmente a via do ácido acético na biossíntese de metabólitos especiais. Dahlgren ${ }^{25}$ coloca a família dentro do grupo angiospérmico derivado, produtor de iridoides.

Bignoniaceae destaca-se tanto pelos números de ocorrência quanto pela diversificação de tipos terpenoídicos. Esse fato está de acordo com o status evolutivo da família, com uma grande expressão da via do acido acético. Foram registrados para essa família: esteróis $(\mathrm{NO}=37)$, triterpenos pentacíclicos $(\mathrm{NO}=52)$, diterpenos $(\mathrm{NO}=1)$, monoterpenos não iridoídicos $(\mathrm{NO}=5)$ e iridoides $(\mathrm{NO}=224)$. Fica evidente a expressiva exploração da via do acetato-mevalonato por essa família e sua grande especialização na produção de derivados iridoídicos.

A química de iridoides é bem diversificada, ocorrendo predominantemente iridoides carbocíclicos $(\mathrm{NO} \%=61,91)$, secoiridoides $(\mathrm{NO} \%=3,44)$ e alcaloides iridoídicos $(\mathrm{NO} \%=7,94)$. Muito embora seja baixa, a ocorrência de secoiridoides desperta a atenção, pois não haviam sido incluídos nessa taxa em estudos anteriores sobre a evolução de iridoides em Angiospermae. ${ }^{26}$

As quinonas são a segunda classe de metabólitos secundários, em número de ocorrência, dentro da família Bignoniaceae (NO = 151). Esta vasta quantidade de registros deve-se ao fato de muito estudo ter sido feito na enorme busca pela naftoquinona lapachol, isolada inicialmente da espécie Handroanthus impetiginosus, sendo muito citada por suas propriedades anticancerígenas, ${ }^{27} \mathrm{o}$ que colocou o ipê roxo no elenco das plantas ameaçadas de extinção. Dentre as quinonas encontradas, 94,04\% são naftoquinonas $(\mathrm{NO}=142)$ e 9 antraquinonas.

Os derivados da via do ácido chiquímico são menos expressivos em Bignoniaceae e não se observa uma grande variedade de tipos estruturais dos seus representantes. Só foram encontrados derivados aromáticos especiais $(\mathrm{NO}=194)$. Dentre esses ocorrem lignanas $\left(\mathrm{C}_{6}-\mathrm{C}_{3}\right)_{2}(\mathrm{NO}=27)$, derivados do ácido cinâmico $\left(\mathrm{C}_{6}-\mathrm{C}_{3}\right)(\mathrm{NO}=69)$, derivados de acetofenona $\left(\mathrm{C}_{6}-\mathrm{C}_{2}\right)(\mathrm{NO}=53)$ e derivados do ácido benzóico $\left(\mathrm{C}_{6}-\mathrm{C}_{1}\right)(\mathrm{NO}=45)$.

A química flavonoídica de Bignoniaceae é dominada por flavonoides de esqueleto regular, não havendo ocorrência de tipos mais especializados, tais como isoflavonoides e neoflavonoides. As 
principais ocorrências de flavonoides na família concentram-se entre flavonas $(\mathrm{NO}=54)$, flavonóis $(\mathrm{NO}=23)$ e di-hidroflavonas $(\mathrm{NO}=$ 18). Ocorrem ainda antocianinas $(\mathrm{NO}=6)$, flavan-3,4-dióis $(\mathrm{NO}=$ 3) e chalcona $(\mathrm{NO}=1)$.

A família Bignoniaceae mostra uma preferência na produção de flavonas o que, segundo Bate-Smith, ${ }^{28}$ pode ser indicativo de posicionamento mais derivado para o táxon.

O método de proteção das hidroxilas flavonoídicas utilizado na família é a proteção por glicosilação $(\mathrm{NO}=41)$, seguido pela proteção por metilação $(\mathrm{NO}=35)$. Bignoniaceae também apresenta hidroxilas desprotegidas $(\mathrm{NO}=18)$ e aquelas com proteção dupla $(\mathrm{NO}=8)$.

A análise dos parâmetros de lenhosidade para Bignoniaceae forneceu um valor de $\mathrm{IH}=38,47$, o que dá uma indicação de hábito de arbustos sobre árvores. Este valor reforça certa lignificação do táxon. Entretanto, o cálculo da razão fo/fl = 2,35 explica a predominância de uma química mais derivada para a família. Segundo Soares e Kaplan, ${ }^{29}$ as famílias lignificadas $(\mathrm{IH} \leq 50,0)$ possuem valores de fo/fl $<1,00$. Esses mesmos autores observaram a ocorrência de famílias lenhosas com valores altos de fo/fl e citaram que, possivelmente, esse fenômeno ocorre em famílias derivadas com retenção de lenhosidade ou que manifestam "secundariamente" essa lenhosidade, isto é, famílias lenhosas que derivam de famílias mais herbáceas.

\section{Perfil químico dos grupos de Bignoniaceae}

A família é dividida em grandes grupos com base, principalmente, em análises de sequência de DNA de cloroplastos ( $\mathrm{rbcL}$, ndhF, trnL-F), sendo eles Bignonieae, Catalpeae, Jacarandeae, Oroxyleae, Tourrettieae, Tecomeae, Clado Paleotropical, Tabebuia alliance e Bignoniaceae unassigned to Clades. ${ }^{10}$

Oito clados são reconhecidos como tribos (Bignonieae, Catalpeae, Coleeae, Crescentieae, Jacarandeae, Oroxyleae, Tecomeae, Tourrettieae) e clados adicionais são nomeados informalmente. Jacarandeae e Catalpeae foram ressuscitados, o primeiro é grupo-irmão para o resto da família. A tribo Eccremocarpeae está incluída no grupo Tourrettieae. Classificações anteriores reconhecem uma Tecomeae grande, porém parafilética com relação a todas as outras tribos. Tecomeae então foi reduzida a um clado de cerca de 12 gêneros com distribuição mundial em ambos os ecossistemas, temperado e tropical. Dois grandes clados, Bignonieae e Crescentiina, respondem por mais de $80 \%$ das espécies da família. As tribos Coleeae e Crescentieae são incluídas em clados maiores, Clado Paleotropical e Tabebuia alliance, respectivamente, sendo que cada grupo inclui uma série de taxa atribuída à tribo Tecomeae tradicional. ${ }^{10}$

A Tabela 1 mostra a distribuição dos metabólitos especiais encontrados para a família Bignoniaceae.

Comparando os resultados aqui encontrados para os grupos de Bignoniaceae com o perfil elaborado por Cipriani, ${ }^{22}$ pode-se afirmar que a química corroborou e muito com a nova circunscrição da família. A antiga tribo Tecomeae era aquela que continha a maior quantidade de quinonas na família. Isso era resultado da grande quantidade de estudos buscando o lapachol nos gêneros Tabebuia e afins. Com a divisão de Tabebuia em três gêneros e o seu deslocamento, junto com alguns outros gêneros antes pertencentes a Tecomeae, para o grupo Tabebuia alliance e Clado Paleotropical, a atual tribo Tecomeae não apresenta mais quinonas.

\section{Jacarandeae}

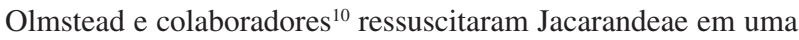
circunscrição incluindo apenas Jacaranda e Digomphia. Essa tribo é irmã do restante de Bignoniaceae. Jacarandeae inclui cerca de 55 espécies que são amplamente distribuídas em toda a região neotropical.
Tabela 1. Distribuição dos metabólitos especiais encontrados para a família Bignoniaceae. Big, Bignonieae; Cat, Catalpeae; Jac, Jacarandeae; Oro, Oroxyleae; Tec, Tecomeae; Tou, Tourrettieae; Pal, Clado Paleotropical; Tab,Tabebuia alliance; BUC, Bignoniaceae unassigned to clades; NO, Número de ocorrência; NOT, Número de ocorrência total; TER, Terpenoides; DCLA, Derivados de cadeia longa da via do acetato; QUI, Quinonas; ALC, Alcaloides; DArE, Derivados aromáticos especiais e FLA, Flavonoides

\begin{tabular}{cccccccc}
\hline Família & \multicolumn{7}{c}{ Número de ocorrência (NO) } \\
Grupos & NOT & TER & DCLA & QUI & ALC & DArE & FLA \\
\hline Big & 146 & 78 & 0 & 10 & 3 & 27 & 28 \\
Cat & 68 & 16 & 0 & 31 & 0 & 18 & 3 \\
Jac & 19 & 7 & 2 & 2 & 0 & 5 & 3 \\
Oro & 50 & 7 & 11 & 2 & 1 & 8 & 21 \\
Tec & 143 & 85 & 5 & 0 & 26 & 34 & 13 \\
Tou & 5 & 5 & 0 & 0 & 0 & 0 & 0 \\
Pal & 227 & 53 & 84 & 66 & 6 & 84 & 15 \\
Tab & 124 & 36 & 16 & 40 & 0 & 14 & 18 \\
BUC & 42 & 32 & 0 & 0 & 2 & 4 & 4 \\
Bignoniaceae & 844 & 319 & 37 & 151 & 38 & 194 & 105 \\
\hline
\end{tabular}

O perfil químico da tribo Jacarandeae é caracterizado por terpenoides $(\mathrm{NO}=7)$, naftoquinonas $(\mathrm{NO}=2)$ e derivados aromáticos especiais $(\mathrm{NO}=5)$. Também ocorrem, porém em menores quantidades, flavonoides $(\mathrm{NO}=3)$ e derivados de cadeia longa da via do ácido acético $(\mathrm{NO}=2)$.

Percebe-se que a tribo apesar de ter a química caracterizada por terpenoides, esses são representados por triterpenos e esteroides, não ocorrendo iridoides, marcador característico da família Bignoniaceae. Isso talvez se deva ao fato dessa tribo apresentar traços ancestrais da família e estar mais relacionada a outras famílias de Lamiales. ${ }^{10}$ Cabe, porém, lembrar que essa tribo ainda é pouco estudada do ponto de vista químico $(\mathrm{NO}=19)$.

A biossíntese dos metabólitos secundários de Jacarandeae baseia-se, principalmente, na rota do ácido acético $(\mathrm{NO}=11)$, sendo a relação chiquimato/acetato igual a 0,45 . A razão fo/fl $=1$ está dentro do esperado para grupos onde o teor de lignificação é alto.

\section{Tourrettieae}

Os dois gêneros de pequenas plantas sublenhosas para trepadeiras herbáceas, distribuídas nos Andes que compõem este pequeno clado foram atribuídos para separar tribos monogenéricas nos tratamentos recentes de Bignoniaceae. ${ }^{8,11}$ Além do hábito semelhante e distribuição, ambos os gêneros têm folhas duplamente compostas (bipinadas para tripinadas em Eccremocarpus e biternada em Tourrettia) com gavinhas ramificadas derivadas de folíolos modificados e as flores sem estaminódios. Dado o forte apoio ao seu caráter monofilético, faz sentido eliminar a redundância de tribos monogenéricas e uní-los em uma única tribo, Tourrettieae, reconhecendo, assim, a sua semelhança e compartilhada ancestralidade. ${ }^{10}$ Tourrettieae compartilha alguns traços com Jacarandeae, que os separam das outras Bignoniaceae.

Das espécies pertencentes a essa tribo, somente uma, Eccremocarpus scaber Ruiz \& Pav., apresenta algum tipo de estudo do ponto de vista químico. Para tanto, o perfil químico da tribo Tourrettieae foi baseado nos metabólitos encontrados para essa espécie.

A tribo mostra preliminarmente um perfil caracterizado por terpenoides $(\mathrm{NO}=5)$, sendo que todos os representantes são iridoides carbocíclicos, dois $\mathrm{C}_{10}$ - e três nor- $\mathrm{C}_{11}$-iridoide.

Com esses poucos dados pode-se dizer que a tribo Tourrettieae 
apresenta até agora uma química totalmente baseada na rota do ácido acético seguindo a tendência geral da família, embora seu $\mathrm{IH}=50,0$ seja indicativo de hábito de fronteira com representantes arbustivos.

\section{Tecomeae}

A maioria dos tratamentos recentes de Bignoniaceae ${ }^{8,11}$ reconhece uma tribo Tecomeae grande e diversificada que inclua todos os táxons com frutos deiscentes, bilocular, cuja deiscência é perpendicular ao septo. Os resultados Olmstead e colaboradores ${ }^{10}$ incluem maior amostragem de Tecomeae l.s. e confirmam a parafilia deste grupo. Eles então circunscreveram uma tribo Tecomeae bem mais reduzida com 12 gêneros e cerca de 55 espécies, distribuídas em todo o mundo.

O perfil químico da tribo Tecomeae é caracterizado por terpenoides $(\mathrm{NO}=85)$, derivados aromáticos especiais $(\mathrm{NO}=34)$ e alcaloides $(\mathrm{NO}=26)$. Também ocorrem, porém em menores quantidades, flavonoides $(\mathrm{NO}=13)$ e derivados de cadeia longa da via do ácido acético $(\mathrm{NO}=5)$ (Figura 2).

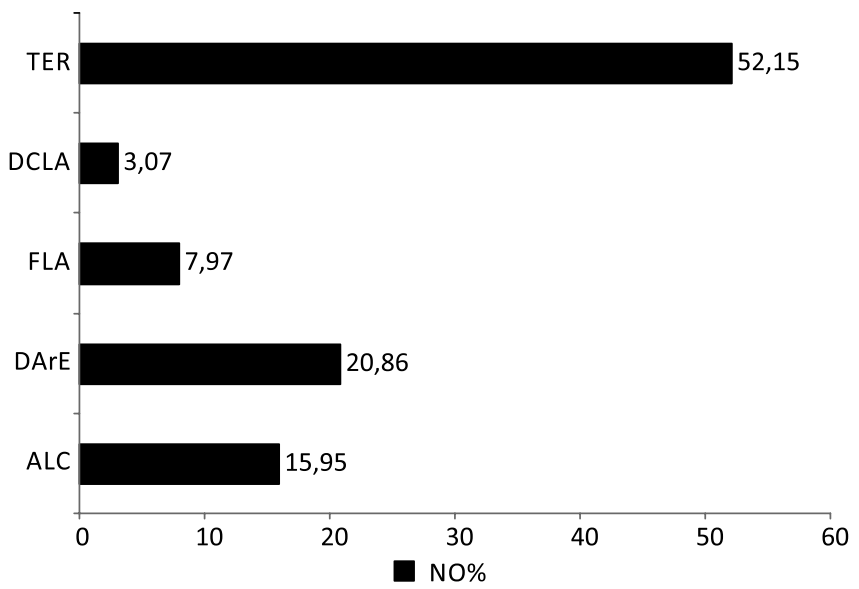

Figura 2. Percentagens dos números de ocorrência (NO\%) de metabólitos especiais encontrados em Tecomeae. ALC $(N O=26) ; D A r E(N O=34) ; F L A$ $(N O=13) ; D C L A(N O=5)$ e TER $(N O=85)$

A biossíntese dos metabólitos secundários de Tecomeae baseia-se, principalmente, na rota do ácido acético $(\mathrm{NO}=116)$, sendo a relação chiquimato/acetato igual a 0,29 .

Com a nova circunscrição da família, a tribo Tecomeae deixou de ser a maior produtora de quinonas, ${ }^{22}$ uma vez que os gêneros responsáveis por essa produção foram deslocados para outros grupos.

Dentro da família Bignoniaceae, a tribo Tecomeae é aquela que apresenta a maior química terpenoídica $(\mathrm{NO}=85)$. Ela caracteriza-se principalmente pela ocorrência de iridoides $(\mathrm{NO}=49)$, sendo também encontrados triterpenos pentacíclicos $(\mathrm{NO}=9)$, esteróis $(\mathrm{NO}=2)$ e alcaloides iridoídicos $(\mathrm{NO}=25)$. Com exceção de duas ocorrências encontradas para Bignoniaceae unassigned to clades, este é o único registro desses derivados nitrogenados para a família. A classe Iridoides é um verdadeiro marcador taxonômico para a família Bignoniaceae e para a tribo Tecomeae. A grande quantidade de iridoides pode servir para corroborar o posicionamento dessa tribo como grupo-irmão de grande parte do restante da família e o seu distanciamento de Jacarandeae, uma vez que nesta não foram encontrados iridoides. ${ }^{10}$

A tribo Tecomeae também se destaca pela presença de alcaloides (NO $=26$ ), onde um é alcaloide derivado do Ciclo de Krebs e 25 são alcaloides iridoídicos. Este o único registro de alcaloides para Bignoniaceae, junto aos dois encontrados em Astianthus viminalis (Kunth) Baill. pertencentes ao grupo das Bignoniaceae unassigned to Clades.
Em relação aos derivados aromáticos especiais, os derivados do ácido cinâmico $(\mathrm{NO}=17)$ e os derivados de acetofenona $(\mathrm{NO}=7)$ são os mais representativos. Ocorrem também derivados do ácido benzoico $(\mathrm{NO}=4)$ e lignanas $(\mathrm{NO}=6)$.

A química flavonoídica da tribo Tecomeae é composta principalmente por flavonas $(\mathrm{NO}=7)$. Ocorrem também flavonóis $(\mathrm{NO}=2)$, antocianinas $(\mathrm{NO}=2)$ e di-hidroflavonas $(\mathrm{NO}=2)$. A razão fo/ $\mathrm{fl}=3,5$ para a tribo segue o padrão da família, onde apesar do grau de lignificação ser alto, a razão fo/fl também apresenta um valor acima de 1 .

Segundo Cipriani e colaboradores, ${ }^{22}$ a tribo Tecomeae era aquela com o maior número de ocorrências dentre as tribos $(\mathrm{NO}=740)$, porém com a nova circunscrição, seus gêneros foram distribuídos entre Tecomeae, Paleotropical clade e Tabebuia alliance. Os gêneros produtores de quinonas ficaram divididos entre os dois últimos grupos.

\section{Clado Paleotropical}

Esse clado contém cerca de 20 gêneros com aproximadamente 150 espécies de árvores . ${ }^{4}$ Além de uma distribuição paleotropical, todos os membros deste clado compartilham hábitos arborescentes distintos para a escalada.

O perfil químico do Clado Paleotropical é caracterizado por terpenoides $(\mathrm{NO}=53)$, quinonas $(\mathrm{NO}=66)$ e derivados aromáticos especiais $(\mathrm{NO}=84)$. Também ocorrem, porém em menor quantidade, flavonoides $(\mathrm{NO}=15)$, alcaloides $(\mathrm{NO}=6)$ e derivados de cadeia longa da via do ácido acético $(\mathrm{NO}=3)$. Este é o grupo mais bem estudado, do ponto de vista químico, dentro da família Bignoniaceae (Figura 3).

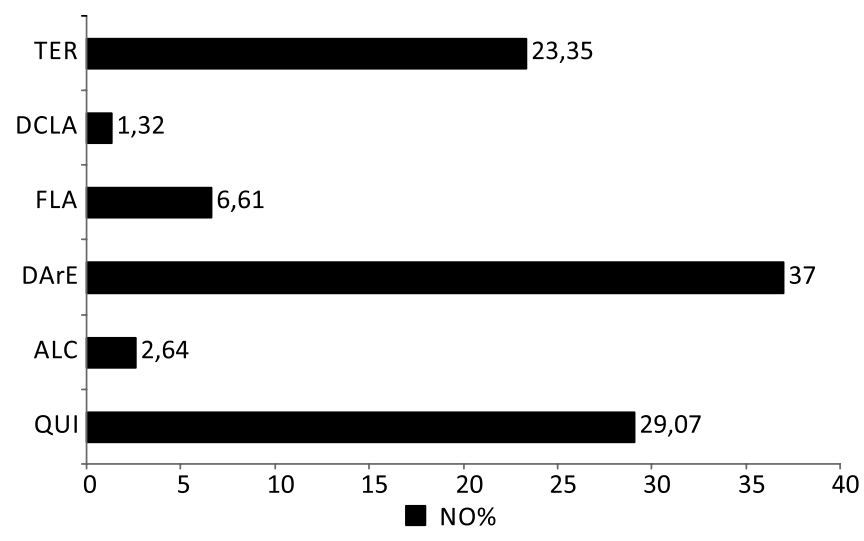

Figura 3. Percentagens dos números de ocorrência (NO\%) de metabólitos especiais encontrados no Clado Paleotropical. QUI $(N O=66) ; A L C(N O$ = 6); DArE $(N O=84) ;$ FLA $(N O=15) ; \operatorname{DCLA}(N O=3)$ e TER $(N O=53)$

A biossíntese dos metabólitos secundários do Clado Paleotropical baseia-se, principalmente, na rota do ácido acético $(\mathrm{NO}=128)$, sendo a relação chiquimato/acetato igual a 0,66.

As quinonas têm grande importância no Clado Paleotropical. De todas as quinonas encontradas para a família Bignoniaceae, $43,71 \%$ pertencem a esse grupo $(\mathrm{NO}=66)$. Dessas, quase todas são naftoquinonas $(\mathrm{NO}=57)$, sendo 9 antraquinonas, as quais são as únicas registradas para a família.

Nesse clado a química terpenoídica é bastante diversificada, destacando-se a presença de monoterpenos não iridoídicos $(\mathrm{NO}=$ 5), o único registro dos mesmos para a família.

Dentre todos os grupos de Bignoniaceae, o Clado Paleotropical tem uma boa representatividade de derivados da via do ácido chiquímico com os derivados aromáticos especiais $(\mathrm{NO}=84)$. Eles representam 43,3\% dos derivados aromáticos especiais encontrados na família. As lignanas presentes neste grupo representam $62,92 \%$ das encontradas na família. 
A química flavonoídica é composta principalmente por flavonas $(\mathrm{NO}=9)$. Ocorrem também flavonóis $(\mathrm{NO}=5)$ e di-hidroflavona $(\mathrm{NO}$ $=1$ ). A razão fo/fl = 1,8 mais uma vez vai de acordo com o perfil da família, cujo grau de lignificação é alto.

\section{Tabebuia alliance}

O clado irmão do Clado Paleotropical é endêmico da região neotropical e contém 14 gêneros. As 147 espécies nesse grupo são todas árvores ou arbustos e partilham a característica das folhas compostas palmadas (com exceção de algumas espécies que apresentam uma redução no número de folíolos e parecem folhas simples). Esse clado é composto em grande parte do gênero Tabebuia l.s. . No entanto, Grose e Olmstead ${ }^{30}$ mostraram que Tabebuia foi uma assembleia parafilética composta por três clados distintos. Assim, eles separaram Tabebuia em três gêneros, Handroanthus, Roseodendron e Tabebuia s.s. ${ }^{31}$

O perfil químico do grupo Tabebuia alliance é caracterizado por terpenoides $(\mathrm{NO}=36)$, quinonas $(\mathrm{NO}=40)$ e derivados de cadeia longa da via do acetato $(\mathrm{NO}=16)$. Também ocorrem flavonoides $(\mathrm{NO}=18)$ e derivados aromáticos especiais $(\mathrm{NO}=14)($ Figura 4$)$.

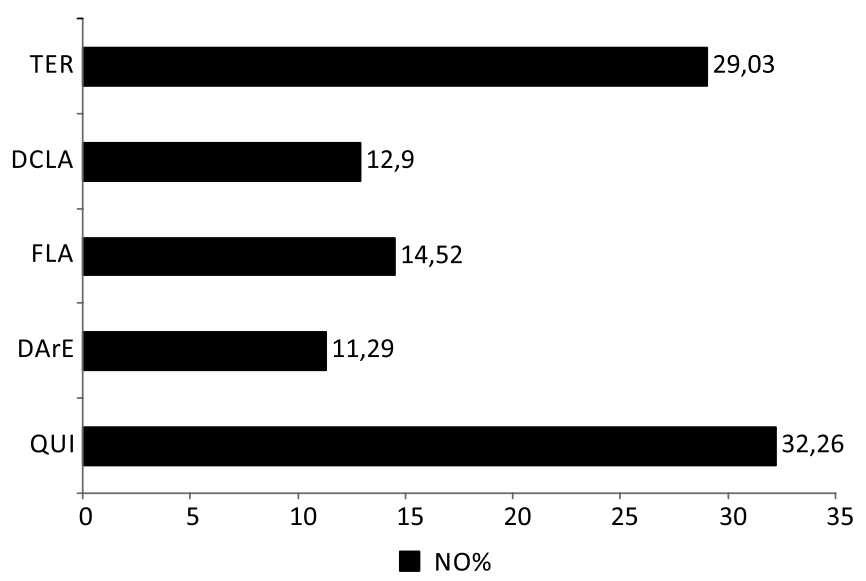

Figura 4. Percentagens dos números de ocorrência (NO\%) de metabólitos especiais encontrados em Tabebuia alliance. QUI $(N O=40) ; D A r E(N O=$ 14); FLA $(N O=18) ; D C L A(N O=16)$ e TER $(N O=36)$

A biossíntese dos metabólitos secundários de Tabebuia alliance baseia-se, principalmente, na rota do ácido acético $(\mathrm{NO}=92)$, sendo a relação chiquimato/acetato igual a 0,15 .

Dentro da Tabebuia alliance a química terpenoídica $(\mathrm{NO}=36)$ caracteriza-se principalmente pela ocorrência de iridoides $(\mathrm{NO}=$ $23)$, sendo também encontrados triterpenos pentacíclicos $(\mathrm{NO}=4)$, esteróis $(\mathrm{NO}=5)$ e secoiridoides $(\mathrm{NO}=4)$. As quinonas representam $32,26 \%$ dos registros para o clado, sendo totalmente constituída de naftoquinonas $(\mathrm{NO}=40)$.

Os derivados da via do ácido chiquímico são representados por derivados do ácido cinâmico $(\mathrm{NO}=5)$, derivados do ácido benzoico $(\mathrm{NO}=5)$ e lignanas $(\mathrm{NO}=4)$.

A química flavonoídica é composta principalmente por flavonas $(\mathrm{NO}=10)$. Ocorrem também flavonóis $(\mathrm{NO}=7)$ e flavan-3,4-diol (NO $=1$ ), sendo este o único registro para a família. A razão fo/fl =1,43.

\section{Oroxyleae}

Este pequeno clado de 4 gêneros e cerca de 6 espécies de árvores e lianas de distribuição Indomalasiana, caracteriza-se por frutos deiscentes paralelamente ao septo (septicidas), uma condição encontrada apenas em Bignonieae neotropicais. ${ }^{8}$

Devido ao fato de apenas dois gêneros da tribo terem sido estudados, o número de ocorrências de metabólitos secundários para Oroxyleae não é muito extenso $(\mathrm{NO}=50)$. O perfil químico de Oroxyleae é caracterizado pela presença principalmente de flavonoides $(\mathrm{NO}=21$ ), ocorrendo também derivados de cadeia longa da via do ácido acético $(\mathrm{NO}=11)$, derivados aromáticos especiais (NO $=8$ ), terpenoides $(\mathrm{NO}=7)$, duas ocorrências de quinonas e uma de alcaloide derivado de ácido aminado alifático.

A química da tribo Oroxyleae leva a um valor da relação chiquimato/acetato igual a 0,38. Essa tribo apresenta o maior percentual de metabólitos especiais derivados de biossíntese mista com os flavonoides $(\mathrm{NO}=21)$ que representam $42 \%$ do total de metabólitos encontrado na tribo.

Dentre os metabólitos biossintetizados pela rota do ácido acético encontram-se naftoquinonas $(\mathrm{NO}=2)$ produzidas em Oroxylum, derivados de cadeia longa da via do acetato $(\mathrm{NO}=11)$, alcaloide $(\mathrm{NO}=1)$ e terpenoides $(\mathrm{NO}=7)$, sendo esses últimos divididos em iridoides carbocíclicos $(\mathrm{NO}=5)$ do tipo bisnoriridoide e esteróis ( $\mathrm{NO}$ =2), todos encontrados em Millingtonia.

Os metabólitos especiais oriundos da rota do ácido chiquímico encontrados para a tribo Oroxyleae são derivados aromáticos especiais $(\mathrm{NO}=8)$, sendo $\mathrm{C}_{6}-\mathrm{C}_{2}(\mathrm{NO}=5), \mathrm{C}_{6}-\mathrm{C}_{3}(\mathrm{NO}=2)$ e $\mathrm{C}_{6}-\mathrm{C}_{1}(\mathrm{NO}=1)$.

Dos metabólitos especiais derivados da rota de biossíntese mista encontram-se os flavonoides $(\mathrm{NO}=21)$, dentre os quais se destacam as flavonas $(\mathrm{NO}=12)$, seguidas pelas di-hidroflavonas $(\mathrm{NO}=6)$, flavonóis $(\mathrm{NO}=2)$ e chalcona $(\mathrm{NO}=1)$, enfatizando que este é o único registro da mesma para a família Bignoniaceae.

Os flavonoides e os derivados de cadeia longa da via do ácido acético são as substâncias mais representativas da tribo Oroxyleae. Chama atenção a relativa abundância dos derivados de cadeia longa da via do acetato que se mostram como verdadeiros marcadores taxonômicos para o grupo.

\section{Catalpeae}

Olmstead e colaboradores ${ }^{10}$ ressuscitaram Catalpeae como um pequeno clado constituído por dois ou três gêneros e aproximadamente 11 espécies.

A grande maioria dos dados químicos encontrados para essa tribo foi referente aos estudos do gênero Catalpa e alguns poucos para Chilopsis. Catalpeae mostra um perfil químico com 68 ocorrências de metabólitos secundários distribuídos em terpenoides $(\mathrm{NO}=16)$, quinonas $(\mathrm{NO}=31)$, derivados aromáticos especiais $(\mathrm{NO}=18)$ e flavonoides $(\mathrm{NO}=3)$ (Figura 5).

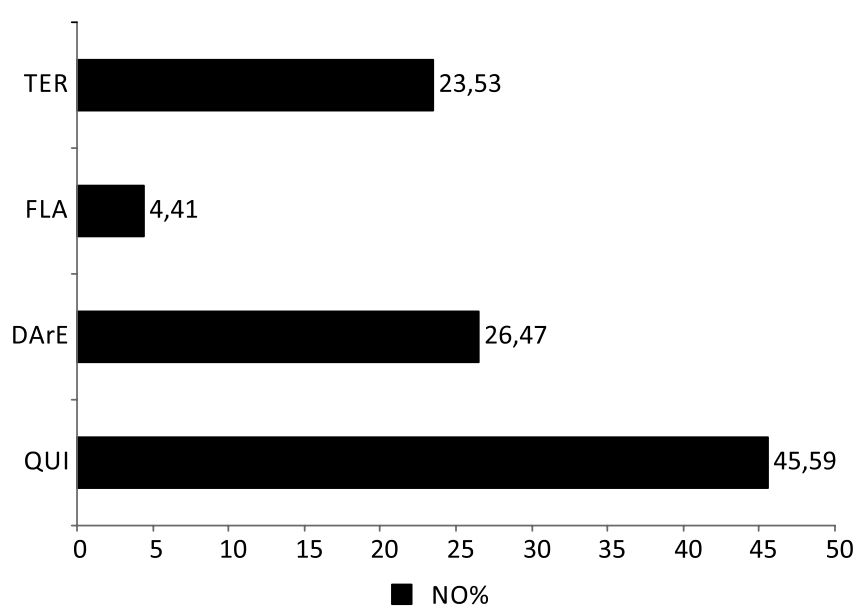

Figura 5. Percentagens dos números de ocorrência (NO\%) de metabólitos especiais encontrados em Catalpeae. QUI $(N O=31) ; D A r E(N O=18) ; F L A$ $(N O=4)$ e TER $(N O=16)$ 
A biossíntese de metabólitos secundários na tribo Catalpeae apóia-se, principalmente, na via do ácido acético $(\mathrm{NO}=47)$ sendo a relação chiquimato/acetato igual a 0,38 .

Os metabólitos derivados da via do ácido acético encontrados foram terpenoides $(\mathrm{NO}=16)$, sendo 15 iridoides carbocíclicos e um secoiridoide, quinonas $(\mathrm{NO}=31)$ do tipo naftoquinona. Desses metabólitos somente um iridoide foi encontrado para o gênero Chilopsis, sendo os demais do gênero Catalpa.

Os representantes da via do ácido chiquímico encontrados para Catalpeae foram derivados aromáticos especiais $(\mathrm{NO}=18)$, sendo um derivado de ácido cinâmico, um derivado de acetofenona e 16 derivados do ácido benzoico.

Os derivados de biossíntese mista $(\mathrm{NO}=3)$ contam com três flavonas.

\section{Bignonieae}

Este grande clado contém 377 espécies e quase a metade das espécies em Bignoniaceae. ${ }^{32} \mathrm{~A}$ tribo neotropical inclui lianas e arbustos que são distribuídos através de uma variedade de habitats, que vão desde as savanas secas até as florestas úmidas. Tradicionalmente, 47 gêneros têm sido reconhecidos na tribo. No entanto, um estudo filogenético detalhado que engloba um terço de todas as espécies Bignonieae indicou que a maioria dos gêneros tradicionalmente reconhecidos não são monofiléticos. A classificação revisada de Bignonieae, reconhecendo apenas grupos monofiléticos diagnosticados por sinapomorfias morfológicas, inclui 21 gêneros. Bignonieae têm sido reconhecidas como um grupo monofilético. A única novidade na circunscrição de Bignonieae é a inclusão de Perianthomega ${ }^{32}$ que tinha sido incluído anteriormente em Tecomeae. ${ }^{11,33}$

O perfil químico da tribo Bignonieae é formado por terpenoides $(\mathrm{NO}=78)$, flavonoides $(\mathrm{NO}=28)$, derivados aromáticos especiais $(\mathrm{NO}=27)$, quinonas $(\mathrm{NO}=10)$ e alcaloides derivados do Ciclo de Krebs $(\mathrm{NO}=3)$ (Figura 6$)$.

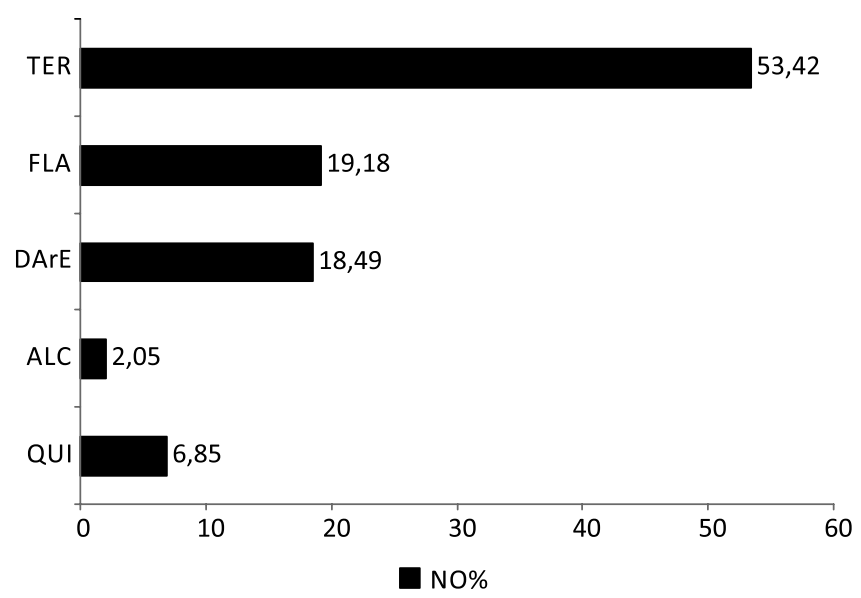

Figura 6. Percentagens dos números de ocorrência (NO\%) de metabólitos especiais encontrados em Bignonieae. QUI $(N O=10) ; A L C(N O=3) ; D A r E$ $(N O=27) ; F L A(N O=28)$ e TER $(N O=78)$

A biossíntese dos metabólitos secundários de Bignonieae baseia-se, principalmente, na rota do ácido acético $(\mathrm{NO}=91)$, sendo a relação chiquimato/acetato de 0,30 .

Os metabólitos todos derivados da via do ácido acético registrados são terpenoides $(\mathrm{NO}=78)$, quinonas $(\mathrm{NO}=10)$, todas naftoquinonas, e alcaloides $(\mathrm{NO}=3)$. A química terpenoídica, a mais bem expressa dentro da tribo, é composta principalmente por iridoides carbocíclicos $(\mathrm{NO}=40)$, triterpenos pentacíclicos $(\mathrm{NO}=25)$. Ocorrem também esteróis $(\mathrm{NO}=12)$ e diterpeno $(\mathrm{NO}=1)$ no gênero Tanaecium $\mathrm{Sw}$.
Os metabólitos oriundos da via do ácido chiquímico são os derivados aromáticos especiais $(\mathrm{NO}=27)$, que se dividem em $\mathrm{C}_{6}-\mathrm{C}_{3}$ $(\mathrm{NO}=10), \mathrm{C}_{6}-\mathrm{C}_{2}(\mathrm{NO}=15)$ e $\mathrm{C}_{6}-\mathrm{C}_{1}(\mathrm{NO}=2)$.

A química flavonoídica da tribo Bignonieae é composta principalmente por flavonas $(\mathrm{NO}=9)$ e di-hidroflavonas $(\mathrm{NO}=8)$. Ocorrem também flavonóis $(\mathrm{NO}=5)$, antocianinas $(\mathrm{NO}=4)$ e flavan-3,4-dióis $(\mathrm{NO}=2)$.

\section{CONCLUSÕES}

Análise dos diferentes parâmetros quimiossistemáticos para o universo de dados reunidos para Bignoniaceae permite considerar que o perfil químico da família se caracteriza pela ocorrência de terpenoides, quinonas, derivados aromáticos especiais e flavonoides, sendo que a biossíntese dos seus metabólitos especiais baseia-se, principalmente, na via do ácido acético, o que está de acordo com o posicionamento da mesma entre as angiospermas mais derivadas. Os iridoides se apresentam como verdadeiros marcadores taxonômicos para a família.

O Índice de Herbacidade encontrado para a família indica o hábito arbustivo dominando sobre árvores, reforçando a lignificação do táxon. Entretanto, o cálculo da razão flavona/flavonol igual a 2,35 explica a predominância de uma química mais evoluída para a família. Isso ocorre em famílias derivadas com retenção de lenhosidade ou que manifestam "secundariamente" essa lenhosidade, isto é, famílias lenhosas que derivam de famílias mais herbáceas.

Dentre os grupos que tiveram investigações do ponto de vista químico, o Clado Paleotropical é o mais estudado, sendo essa química dominada por derivados de cadeia longa da via do ácido acético e derivados aromáticos especiais. Bignonieae também apresenta um grande número de registros sendo as principais ocorrências de terpenoides, juntamente com Tecomeae, cuja química também é caracterizada por terpenoides e Tabebuia alliance, cujo grupo é dominado por quinonas e terpenoides. Catalpeae apresenta uma química caracterizada por, principalmente, quinonas. Oroxyleae já é um grupo menos estudado quimicamente e apresenta, proporcionalmente em relação aos demais, a maior química flavonoídica. Jacarandeae também é pouco estudado e caracterizado por terpenoides e derivados aromáticos especiais. Já o grupo Tourrettieae tem somente uma de suas espécies estudadas do ponto de vista químico e apresentou somente terpenoides.

\section{AGRADECIMENTOS}

À Coordenação de Aperfeiçoamento de Pessoal de Nível Superior (CAPES) e ao Conselho Nacional de Desenvolvimento Científico e Tecnológico $(\mathrm{CNPq})$ pelo suporte financeiro.

\section{REFERÊNCIAS}

1. Angiosperm Phylogeny Group III.; Bot. J. Linn. Soc. 2009, 161, 105.

2. Olmstead, R. G.; Pamphilis, C. W.; Wolfe, A. D.; Young, N. D.; Elisens, W. J.; Reeves, P. A.; Am. J. Bot. 2001, 88, 348.

3. Oxelman, B.; Kornhall, P.; Olmstead, R. G.; Bremer, B.; Taxon 2005, $54,411$.

4. Lohmann, L. G.; Ulloa, C. U.; Bignoniaceae in iPlants prototype checklist [online], 2007, http://www.iplants.org, acessada em Dezembro 2010.

5. Judd, W. S.; Campbelll, C. S.; Kellog, E. A.; Stevens, P. F.; Plant Systematics: A Phylogenetic Approach, Sinauer Associates: Sunderland, 1999.

6. Bremer, K.; Backlund, A.; Sennblad, B.; Swenson, U.; Andreasen, K.; Hjertson, M.; Lundberg, J.; Backlund, M.; Bremer, B.; Plant Syst. Evol. 2001, 229, 137.

7. Bremer, B.; Bremer, K.; Heidari, N.; Erixon, P.; Olmstead, R. G.; Anderberg, A. A.; Kallersjo, M.; Barkhordarian, E.; Mol. Phylogenet. Evol. 2002, 24, 274. 
8. Gentry, A. H.; Bignoniaceae-Part I (Crescentieae and Tourretieae), Flora Neotropica. Monogr. 25, The New York Botanical Garden: New York, 1980.

9. Mabberley, D. J.; The Plant Book - A Portable Dictionary of the Vascular Plant, $2^{\text {nd }}$ ed., Cambridge University Press: Cambridge, 1997.

10. Olmstead, R. G.; Zjhra, M. L.; Lohmann, L. G.; Grose, S. O.; Eckert, A. J.; Am. J. Bot. 2009, 96, 1731.

11. Fischer, E.; Theisen, I.; Lohmann, G. L. Em The families and Genera of Vascular Plants; Kadereit, J. W., ed.; Springer-Verlag: Heidelberg, 2004, vol. VII.

12. Harborne, J. B.; Phytochemistry 1967, 6, 1643.

13. Rohatgi, B. K.; Gupta, R. B.; Roy, D.; Khanna, R. N.; Indian J. Chem. $B$ 1983, 22, 886 .

14. Binutu, O. A.; Lajubutu, B. A.; S. Afr. J. Med. Sci. 1994, 23, 269.

15. von Poser, G. L.; Schripsema, J.; Henriques, A. T.; Jensen, S. R.; Biochem. Syst. Ecol. 2000, 28, 351

16. Alcerito, T.; Barbo, F. E.; Negri, G.; Santos, D. Y. A. C.; Meda, C. I.; Young, M. C. M.; Chávez, D.; Blatt, C. T. T.; Biochem. Syst. Ecol. 2002, 30, 677 .

17. Alguacil, L. F.; Mera, A. G.; Gómez, J.; Llinares, F.; Morales, L.; Munoz-Mingarro, M. D.; Pozuelo, J. M.; Orellana, J. A. V.; J. Ethnopharmacol. 2000, 70, 227.

18. Andrade-Cetto, A.; Heinrich, M.; J. Ethnopharmacol. 2005, 99, 325.

19. Malerich, J. P.; Maimone, T. J.; Elliott, G. I.; Trauner, D.; J. Am. Chem. Soc. 2005, 127, 6276.
20. Blatt, C. T. T.; Santos, M. D.; Salatino, A.; Plant Syst. Evol. 1998, 210, 289.

21. Nicoletti, M.; Serafini, M.; Bianco, A.; Passacantili, P.; Ann. Bot.-Rome 1984, 42,81 .

22. Cipriani, F. A.; Cidade, W. F.; Soares, G. L. G.; Kaplan, M. A. C. Em Abordagem quimiossistemática e evolução química de fanerógamas; Kaplan, M. A. C.; Abreu, H. S.; Lima, H. R. P.; Soares, G. L. G., eds.; Edur: Seropédica, 2010, cap. 3.

23. Gottlieb, O. R.; Kaplan, M. A. C.; Borin, M. R. M. B.; Biodiversidade: Enfoque Químico Biológico do Funcionamento da Natureza, Ed. da UFRJ: Rio de Janeiro, 1996.

24. Cronquist, A.; The Evolution and Classification of Flowering Plants, $2^{\text {nd }}$ ed., The New York Botanical Garden: New York, 1988.

25. Dahlgren, R. M. T.; Bot. J. Linn. Soc. 1980, 80, 91.

26. Sampaio-Santos, M. I.; Kaplan, M. A. C.; J. Braz. Chem. Soc. 2001, 12, 144.

27. Hostettmann, K.; Lea, P. J.; Biologically Active Natural Products. Proceedings of the Phytochemical Society of Europe, Oxford Science Publications/Claredon Press: Oxford, 1987, vol. 27.

28. Bate-Smith, E. C.; Bot. J. Linn.Soc. 1962, 58, 95.

29. Soares, G. L. G.; Kaplan, M. A. C.; Bot. J. Linn. Soc. 2001, 135, 61.

30. Grose, S. O.; Olmstead, R. G.; Syst. Bot. 2007, 32, 650.

31. Grose, S. O.; Olmstead, R. G.; Syst. Bot. 2007, 32, 660.

32. Lohmann, L. G.; Am. J. Bot. 2006, 93, 304.

33. Gentry, A. H.; Ann. Mo. Bot. Gard. 1992, 79, 53. 